\section{Could autologous adipose-derived stromal vascular fraction turn out an unwanted source of profibrotic myofibroblasts in systemic sclerosis?}

With great interest, I read the recent publication by Magalon et $a l^{1}$ in the Annals of the Rheumatic Diseases. The results of this study are placed in the scenario of a quite complex disease featuring vasculopathy, autoimmunity and extensive multiorgan fibrosis whose life-threatening nature is well testified by a 5 -year mortality of $30 \%-50 \%$ in a subset of patients with diffuse cutaneous systemic sclerosis (SSc) and internal organ involvement. ${ }^{2}$ Although substantial basic/translational and clinical research progresses have been achieved over the past decade, current treatments are mainly organ based and do not result in a cure which highlights the urgent need of developing new potentially disease-modifying therapies and personalised medicine approaches. $^{2}$

In this context, the adipose-derived stromal vascular fraction (ADSVF) has recently gained attention as an innovative biotherapy because of its abundance of mesenchymal-like stem/ stromal cells (referred to as adipose-derived stem cells; ADSC), accessibility and ease of harvest. The same research team has previously reported encouraging results from a phase I clinical trial showing a good safety profile and a potential efficacy of local injection of autologous ADSVF to treat hand disability in patients with SSc. ${ }^{3}$ This evidence was further substantiated by an up to 2-year follow-up analysis indicating an improvement of ischaemic vasculopathy (ie, Raynaud's phenomenon and digital ulcers), hand pain and global quality of life without any significant worsening of fibrosis as assessed by modified Rodnan skin score applied to the hands. ${ }^{4}$ The aforementioned observations represented the obvious groundwork for the herein commented study, ${ }^{1}$ in which the authors provided a thorough characterisation of the cellular and molecular profiles of therapeutic-grade ADSVF harvested from patients with SSc in comparison with healthy donors (HD). Such an analysis is of extraordinary importance, especially when considering that the composition of ADSVF is extremely heterogeneous as it includes not only ADSC, that represent its major constituents, but also endothelial cells/ progenitors, pericytes, and haematopoietic and immune cells. ${ }^{1}$ The authors reported no substantial difference in the distribution of the various cell populations in SSc-ADSVF compared with HD-ADSVF, and the two products performed similarly with only slight differences in terms of their vasculogenic/angiogenic capacity assessed by a number of in vitro and in vivo assays. ${ }^{1}$ Nonetheless, global and single-cell RNA-sequencing approaches and analysis of ADSVF-derived secreted factors revealed a differential endothelial and stromal cell molecular signature of the SSc-ADSVF reflecting endothelial activation, deregulation of angiogenesis and fibrosis, such as an upregulation of growth differentiation factor- 15 that has previously been implicated in SSc-related fibrosis. ${ }^{1}$

I particularly appreciated the balanced conclusions of the authors that not only emphasised the potential of autologous ADSVF to treat SSc-related vasculopathy on the basis of its preserved vascular repair capacity, but also frankly acknowledged that the significance of the molecular profile alterations detected in the SSc-ADSVF deserves an in-depth investigation, since they may be relevant for the fibrotic process. ${ }^{1}$ In this regard, I believe that some additional cues are worth considering. First, another study has previously analysed the ADSVF from patients with diffuse cutaneous SSc and reported somehow different results. ${ }^{5}$ In fact, Virzì et $a l^{5}$ have shown that SScADSVF displays an altered cell composition characterised by a reduced number of cells with a stem-like phenotype, as well as a high content of proinflammatory cytokines and a shortage of angiogenic factors. Moreover, at variance with an earlier study showing that isolated SSc-ADSC exhibit similar biological properties (ie, surface antigenic profile, proliferation and differentiation potentials, immunosuppressive properties and capacity to support endothelial cell tube formation) compared with HD-ADSC, ${ }^{6}$ Virzì et al ${ }^{5}$ found that SSc-ADSC may retain high multipotency but fail to sustain terminally differentiated adipocyte, osteocyte and chondrocyte progenies. Second, similarly to previous findings on SSc bone marrow-derived mesenchymal stem/stromal cells, ${ }^{7}$ Lee et $a l^{8}$ have recently reported that early passage SSc-ADSC have a profibrotic and antiadipogenic phenotype characterised by high levels of the myofibroblast marker $\alpha$-smooth muscle actin and low expression of both caveolin-1 and the adipogenic marker FABP4. Of note, they also demonstrated that a myofibroblast-like phenotype could be induced in HD-ADSC by treatment with transforming growth factor- $\beta^{8}$ which suggests that the SSc pathological environment might be relevant in determining an unwanted profibrotic fate of ADSC. Finally, we should not overlook that cell fate mapping studies in the bleomycin-induced mouse model of skin fibrosis clearly demonstrated that adiponectin-positive progenitors that are normally confined to the intradermal adipose tissue compartment redistribute into the lesional dermis, where they lose the adipocytic markers and acquire a myofibroblast-like phenotype. ${ }^{9}$ Taken together, it is clear that the functional experimental approach employed by Magalon et al ${ }^{1}$ could exclusively disclose the vascular repair performance of SSc-ADSVF, but much work is still to be done (eg, xenogeneic human SSc-ADSVF injection in mouse models) to definitely rule out that autologous ADSVF might even behave as an unwanted source of profibrotic myofibroblasts in a therapeutic setting. Based on either the evidence that the specific pathological environment might be crucial in affecting the ADSC fate or the lesson we recently learnt from gene expression profiling studies on the existence of different molecular subtypes of SSc fibrotic skin disease, ${ }^{8} 10$ a future in-depth molecular and functional characterisation of ADSVF in larger SSc cohorts has the great potential to help in predicting which patients are more likely to benefit from an autologous ADSVF-based therapeutic approach.

\section{Mirko Manetti $\odot$}

Correspondence to Dr Mirko Manetti, Department of Experimental and Clinical Medicine, Section of Anatomy and Histology, University of Florence, Florence I-50134, Italy; mirko.manetti@unifi.it

Handling editor Josef Smolen

Contributors MM has completely drafted and finalised the manuscript.

Funding The author has not declared a specific grant for this research from any funding agency in the public, commercial or not-for-profit sectors.

Competing interests None declared.

Patient consent for publication Not required.

Provenance and peer review Not commissioned; internally peer reviewed.

(c) Author(s) (or their employer(s)) 2020. No commercial re-use. See rights and permissions. Published by BMJ.

Check for updates

To cite Manetti M. Ann Rheum Dis 2020;79:e55. 
Received 25 February 2019

Accepted 2 March 2019

Published Online First 13 March 2019

\section{SLinked}

http://dx.doi.org/10.1136/annrheumdis-2019-215327

Ann Rheum Dis 2020:79:e55. doi:10.1136/annrheumdis-2019-215288

ORCID iD

Mirko Manetti http://orcid.org/0000-0003-3956-8480

\section{REFERENCES}

1 Magalon J, Velier M, Simoncini S, et al. Molecular profile and proangiogenic activity of the adipose-derived stromal vascular fraction used as an autologous innovative medicinal product in patients with systemic sclerosis. Ann Rheum Dis 2019;78:391-8

2 Manetti M, Matucci-Cerinic M. The new frontier in systemic sclerosis: from epigenetics to new treatments. Rheumatology 2015;54:1757-8.

3 Granel B, Daumas A, Jouve E, et al. Safety, tolerability and potential efficacy of injection of autologous adipose-derived stromal vascular fraction in the fingers of patients with systemic sclerosis: an open-label phase I trial. Ann Rheum Dis 2015; 74:2175-82.

4 Daumas A, Magalon J, Jouve E, et al. Long-term follow-up after autologous adiposederived stromal vascular fraction injection into fingers in systemic sclerosis patients. Curr Res Trans/ Med 2017;65:40-3

5 Virzi F, Bianca P, Giammona A, et al. Combined platelet-rich plasma and lipofilling treatment provides great Improvement in facial skin-induced lesion regeneration for scleroderma patients. Stem Cell Res Ther 2017:8.

6 Capelli C, Zaccara E, Cipriani P, et al. Phenotypical and functional characteristics of in vitro-expanded adipose-derived mesenchymal stromal cells from patients with systematic sclerosis. Cell Transplant 2017;26:841-54.

7 Hegner B, Schaub T, Catar R, et al. Intrinsic deregulation of vascular smooth muscle and myofibroblast differentiation in mesenchymal stromal cells from patients with systemic sclerosis. PLoS One 2016;11:e0153101.

8 Lee R, Del Papa N, Introna M, et al. Adipose-derived mesenchymal stromal/stem cells in systemic sclerosis: alterations in function and beneficial effect on lung fibrosis are regulated by caveolin-1. J Scleroderma Relat Disord 2019.

9 Marangoni RG, Korman BD, Wei J, et al. Myofibroblasts in murine cutaneous fibrosis originate from adiponectin-positive intradermal progenitors. Arthritis Rheumatol 2015:67:1062-73

10 Martyanov V, Whitfield ML. Molecular stratification and precision medicine in systemic sclerosis from genomic and proteomic data. Curr Opin Rheumatol 2016;28:83-8. 